\title{
La « référentialisation » des compétences à l'école, conceptions et mises en œuvre
}

Des attendus aux malentendus

The referencialization of competences in schools, conception and implementation: from avenues to misunderstandings

La referencialización de las competencias en la escuela, concepciones y puestas en práctica: de las expectativas a los malentendidos

Die Referentialisierung der Kompetenzen in der Schule, Auffassungen und Anwendungen: von den Erwartungen bis zu den Missverständnissen

Jean-Claude Coulet

\section{OpenEdition}

Journals

Édition électronique

URL : http://journals.openedition.org/rechercheformation/199

DOI : 10.4000/rechercheformation.199

ISSN : 1968-3936

Éditeur

ENS Éditions

Édition imprimée

Date de publication : 1 juillet 2010

Pagination : 47-62

ISSN : 0988-1824

Référence électronique

Jean-Claude Coulet, «La « référentialisation » des compétences à l'école, conceptions et mises

en œuvre », Recherche et formation [En ligne], 64 | 2010, mis en ligne le 01 juillet 2012, consulté le 19 avril 2019. URL : http://journals.openedition.org/rechercheformation/199; DOI : 10.4000/ rechercheformation. 199 


\title{
La "référentialisation" des compétences à l'école, conceptions et mises en œuvre
}

\section{Des attendus aux malentendus}

\author{
$>$ Jean-Claude COULET \\ Université Rennes 2-Haute-Bretagne, CRPCC (Centre de recherches en psychologie, \\ cognition et communication, EA 1285)
}

\begin{abstract}
RÉSUMÉ - À partir d'une recherche, analysant l'activité de professeurs sur la base d'un modèle fonctionnel de la compétence, l'article vise à montrer, au-delà des débats suscités par l'approche par compétences, comment la formulation des référentiels du ministère de l'Éducation nationale risque fort d'accentuer certains malentendus relatifs à la dialectique du " réussir " et du " comprendre " dans les apprentissages des élèves. Quelques pistes de réflexion sont ensuite proposées en vue d'instrumentaliser ces référentiels, au-delà de leur aspect normatif, au service d'une capitalisation des compétences professionnelles des enseignants.
\end{abstract}

MOTS-CLÉS・ norme, compétence, apprentissage, tutorat

À l'image de ce qui se fait dans bien d'autres pays (Canada, États-Unis, Suisse, Pays-Bas, Royaume-Uni, Belgique...) et dans une logique que certains n'hésitent pas à soupçonner d'être inspirée par celle qui prévaut dans les entreprises (Hirtt, 2009), la France est entrée dans une phase de " référentialisation " des compétences à l'école. Celle-ci consiste à définir les compétences attendues en répertoriant, de manière distincte, les connaissances, capacités et attitudes, supposées en constituer l'essence 1 . Sont ainsi visées les compétences des élèves, explicitées dans le " socle commun de connaissances et de compétences " (BO, n ${ }^{\circ} 29$ du 20 juillet 2006) mais, également, les compétences professionnelles que doivent développer et mettre en œuvre les professeurs (BO, $\mathrm{n}^{\circ} 1 \mathrm{du} 4$ janvier 2007). Une telle focalisation sur la notion de compétence, n'a pas manqué de faire émerger de multiples

1 Dans une trilogie semblable, la recommandation du Parlement européen et du Conseil, du 18 décembre 2006 sur les compétences clés pour l'éducation et la formation tout au long de la vie, retient les termes de : connaissances, aptitudes et attitudes, Journal officiel L 394/10 du 30 décembre 2006, disponible sur Internet : <http://eur-lex.europa.eu/LexUriServ/LexUriServ.do?uri=0]:L:2006:394:0010:0018:fr:PDF>, consulté le 16 août 2010. 
commentaires et controverses autour d'enjeux explicites et implicites associés à cette démarche. Toutefois, au-delà de la pertinence des arguments avancés par les uns et les autres dans l'approche par compétences (APC), n'est-ce pas la conception même de compétence qu'il faudrait interroger?

Ce texte a pour objectif de défendre cette thèse, tant d'un point de vue théorique qu'à travers l'exemple d'une recherche analysant l'activité concrète de professeurs en situation de classe (Coulet, 2007b) à partir d'un modèle alternatif de la compétence (Coulet, 2007a ; Coulet, à paraître). À la lumière des résultats de cette étude, on tentera de montrer, comment les référentiels développés par le ministère de l'Éducation nationale (MEN), au-delà des potentialités qu'ils portent, risquent fort d'accentuer certains malentendus relatifs à la dialectique du " réussir " et du " comprendre "2 (Piaget, 1974 ; Fabre, 1997 ; Perrenoud, 2002 ; Lenoir \& Pastré, 2008) dans les apprentissages réalisés par les élèves, sans nécessairement armer les enseignants des outils conceptuels et pratiques dont ils auraient besoin pour éviter ce travers. Par ailleurs, nous nous efforcerons de dégager quelques pistes de réflexion, susceptibles de minorer la fonction normative de ce type de référentiels afin d'en faire de véritables outils de dynamisation de l'évolution des compétences.

\section{Usages de la compétence et enjeux associés}

Au cours des dernières décennies, la notion de compétence s'est imposée comme centrale dans un grand nombre de pratiques sociales. En lien, fonctionnel ou circonstanciel, avec les mutations ou évolutions qui se sont produites dans les organisations (Oiry, 2005), elle a diffusé jusqu'à l'école via, notamment, la formation professionnelle.

Au sein des organisations, elle a changé le rapport aux qualifications professionnelles en plaçant les salariés en situation de responsabilité (Lichtenberger, 1999) quant à leur employabilité sur la base des compétences qu'ils ont à acquérir, entretenir, améliorer, diversifier (Stroobants, 1998). La pratique du bilan de compétences, mise en place en France par la loi $^{3}$, au début des années 1990, s'inscrit dans cette logique, tant dans une perspective d'accès ou de retour à l'emploi que de mobilité professionnelle ou de gestion de carrière (Gaudron \& Croity-Belz, 2005). De même, la Validation des acquis de l'expérience (VAE) ${ }^{4}$ vise à promouvoir des compétences individuelles, cette fois, en termes de diplômes, pour favoriser

2 Piaget (1974) insiste sur l'autonomie de l'action (qui permet la réussite) et dont la conceptualisation (compréhension) résulte d'une prise de conscience ultérieure. De nombreuses approches pédagogiques sont discutées en référence à ce cadre théorique (Fabre, 1997 ; Perrenoud, 2002 ; Lenoir \& Pastré, 2008).

3 Loi 91-1405 du 31 décembre 1991.

4 Loi 2002-73 du 17 janvier 2002.

Cf. également, le rapport du groupe de travail sur la VAE, présidé par Vincent Merle, professeur au CNAM, et remis, en décembre 2008, au secrétaire d'État à l'emploi, disponible sur Internet : <http://www.cariforef-mp. asso.fr/files/vae/Extranet-VAE/rapport_groupe_travail_vae_merle_2009.pdf>, consulté le 25 août 2010. 
le parcours professionnel de personnes également censées être responsables de leurs choix.

Dans le champ de l'éducation et de la formation (Perrenoud, 1999 ; Crahay, 2006 ; Jonnaert, 2006 ; Carette, 2007), la notion de compétence est devenue tout aussi cruciale, qu'il s'agisse de la définition des objectifs pédagogiques, de l'élaboration des programmes, de l'évaluation des élèves ou, encore, de la professionnalisation des enseignants.

L'ensemble de ces évolutions tend donc à faire de la compétence la composante essentielle de l'articulation entre formation et emploi. Pour autant, les positions sont loin d'être unanimes sur la notion même de compétence et pas davantage quant à la manière dont doit être conçue cette articulation. Ceci peut expliquer la vivacité du débat autour de la notion et l'importance des enjeux qui lui sont attachés. Notre propos n'est pas de discuter ici l'ensemble des points de vue avancés. Cependant, comme le montre bien Hirtt (2009), on peut considérer que l'essentiel de la polémique porte, d'une part, sur l'assujettissement, ou non, de la formation des individus aux logiques socioéconomiques organisant le marché du travail et, d'autre part, sur la place faite aux savoirs académiques dans les pratiques pédagogiques. Ainsi, pour Hirtt, l'APC, dans le champ de l'éducation et de la formation, serait une façon de finaliser les apprentissages en termes d'activités performantes telles qu'elles sont requises par l'emploi, au détriment des savoirs académiques considérés comme seuls garants d'une intelligibilité critique du monde. Au contraire, pour ses adeptes, l'APC serait une manière de rendre vivants ces savoirs académiques, grâce à leur mobilisation adaptée en situation, pour faire face à telle ou telle classe de tâches.

On le voit, posée en ces termes, l'opposition apparaît difficilement surmontable. À y regarder de plus près, on peut néanmoins se demander si la cristallisation des positions ne se niche pas dans la conception même de compétence, sous-jacente aux arguments avancés.

\section{Les conceptions de la compétence}

Indiscutablement, la notion de compétence souffre de définitions multiples et très hétérogènes en fonction de leurs courants théoriques d'origine mais aussi, d'un certain consensus la présentant comme le résultat d'un assemblage d'éléments divers. Ainsi, de façon récurrente, la compétence est décrite comme constituée de savoirs, de savoir-faire et de savoir-être : une trilogie déjà ancienne et déclinée, plus récemment, en termes de combinaison de connaissances, de capacités et d'attitudes (ministère de l'Éducation nationale ${ }^{5}$ ). Dès lors, deux difficultés principales émergent.

5 Cf. le décret $\mathrm{n}^{0}$ 2006-830 du 11-7-2006 concernant le socle commun de connaissances et de compétences. 
La première difficulté est à mettre en rapport avec le béhaviorisme, qui a largement inspiré (ou justifié), au-delà de la pédagogie par objectifs ${ }^{6}$, une conception de la compétence se focalisant sur les seuls résultats de l'activité, c'est-à-dire sur des comportements mesurables exprimés dans une performance. Dans ce cas, les adversaires de l'APC ont beau jeu de dénoncer le pilotage de la formation des individus par les résultats de l'activité devant être, en dernier ressort, efficace au regard des attentes socioéconomiques, sans se préoccuper des processus engendrant cette activité et, notamment, du rôle des savoirs académiques considérés au mieux comme des ressources à disposition pour agir efficacement. Évidemment, une approche aussi radicale pourrait conduire à penser qu'elle est relativement marginale, mais il faut bien reconnaître qu'elle reste encore assez souvent invoquée, en particulier dans le domaine professionnel, au nom d'un certain pragmatisme. Elle peut également représenter, dans certains cas, une manière plus acceptable d'exprimer un point de vue innéiste puisque, ne pas avoir à s'interroger sur les processus de mobilisation et de construction des ressources pour agir efficacement, offre la possibilité de les concevoir comme l'expression de "talents naturels". En outre, ces positions béhavioristes ou innéistes sont d'autant plus séduisantes que l'approche en termes de juxtaposition ou de combinaison de savoirs, savoir-faire et savoir-être, n'est pas forcément très satisfaisante pour rendre compte des processus en jeu dans la mobilisation et la construction des compétences.

L'autre difficulté est précisément liée à cette approche taxinomique ou " nominaliste" (Crahay, 2006) des éléments constitutifs de la compétence. En effet, si la rupture avec la théorie béhavioriste associée à la pédagogie par objectifs (Monchatre, 2008) est clairement affirmée par les tenants de l'APC, rendre compte des processus en jeu dans la production de la performance, en se limitant à distinguer des savoirs, savoir-faire et savoir-être reste évidemment très problématique. Cela est d'autant plus vrai, comme le souligne Bellier (1999, p. 234), qu'on ne dispose pas d'une " théorie construite qui expliquerait pourquoi la compétence comprend ces éléments ". C'est également ce que dénonce Crahay (2006, p. 105) à propos de l'APC, Iorsqu'il écrit : " il serait plus utile de se doter d'un modèle conceptuel que de s'accrocher à un mot ". Quant à Monchatre (2008, p. 38), elle affirme : " un rapide examen des principales réflexions sur la compétence en pédagogie permet en effet de voir que cette notion se caractérise par une grande fragilité sur le plan théorique ". En outre, cette juxtaposition d'éléments constitutifs de la compétence conduit, le plus souvent, à perdre de vue l'idée même de processus et à traiter chaque composante ainsi avancée pour elle-même. C'est très exactement ce qui se passe pour le socle commun de connaissances et de compétences (cf. annexe du décret $n^{\circ}$ 2006-830 du 11-7-2006). En effet, après avoir défini la compétence comme " une combinaison de connaissances fondamentales pour notre temps, de

6 Cf. Monchatre (2008) à propos des filiations (conception des objectifs pédagogiques) et ruptures (théoriques) entre Pédagogie par objectifs (PPO) et Approche par compétence (APC). 
capacités à les mettre en œuvre dans des situations variées, mais aussi d'attitudes indispensables tout au long de la vie ", le texte énumère, de façon indépendante, les connaissances, capacités et attitudes relatives à chacune des sept compétences constituant le socle $\mathrm{e}^{7}$. Les tenants de l'APC ont alors de bons arguments pour montrer que les savoirs académiques ne sont pas oubliés! Cependant, la question de l'exploitation de telles listes (Savoyant, 1999) reste posée.

Ainsi, à défaut d'un modèle théorique, exprimant fonctionnellement la manière dont la compétence structure l'activité en situation jusque dans ses formes de régulation, dont dépendent les apprentissages (Chauvigné \& Coulet, à paraître), on risque fort de laisser perdurer une vaine confrontation de points de vue inconciliables.

\section{Pour aller plus loin : un exemple d'analyse de pratiques de classe}

Notre engagement sur cette voie, outre l'élaboration d'un modèle théorique (Coulet, à paraître) de la compétence $\left(\mathrm{MADDEC}^{8}\right)$ et d'un modèle de tutorat $\left(\mathrm{MADIC}^{9}\right)$, présentés succinctement ci-dessous, nous a conduit à mener des recherches orientées dans plusieurs directions.

L'exemple, exposé brièvement ici, est celui d'une recherche ${ }^{10}$ visant à caractériser, chez deux enseignants, maîtres formateurs de cours préparatoire, les formes de guidage des élèves qu'ils mettent en œuvre au cours de deux séances de classe en mathématiques. Dans le cadre de leur préparation, les deux enseignants, aidés par un chercheur de l'équipe ${ }^{11}$, se sont entendus sur les contenus à aborder. II s'agit, d'une approche de la division à partir de situations de quantification de groupements donnés (ex : combien de bandes de cinq étoiles sont nécessaires pour disposer x étoiles sur un sapin de Noël). Par ailleurs, des épreuves de pré-test et de post-test ont été administrées aux élèves des deux classes concernées afin de mesurer leurs acquisitions et tenter de les mettre en rapport avec les formes de guidage dont ils ont bénéficié, de la part de leur enseignant, au cours des deux séances.

Dans cette situation d'enseignement-apprentissage, sont en jeu deux types de compétences : celles des élèves confrontés à cette classe de tâches et celles des enseignants quant à leur manière de guider les apprentissages des élèves.

7 L'énoncé des dix compétences professionnelles des enseignants ( $B 0 n^{0} 1$ du 4 janvier 2007) n'échappe pas à cette critique.

8 Modèle d'analyse dynamique pour décrire et évaluer les compétences.

9 Modèle d'aide au développement individuel des compétences.

10 Cette recherche (cf. également, Coulet, 2007b) est une contribution à un projet PIREF plus global concernant les pratiques enseignantes et la réussite des élèves (Sensevy, 2007).

11 Il s'agissait, pour lui, de veiller à ce que l'accord soit bien explicité et approprié par chacun des enseignants. 
Sur le plan théorique, nous définissons la compétence comme " une organisation dynamique de l'activité, mobilisée et régulée par un sujet pour faire face à une tâche donnée, dans une situation déterminée ". En référence à un ensemble de travaux, de psychologie du développement cognitif ou portant sur la résolution de problèmes, nous l'avons modélisée, pour cette recherche ${ }^{12}$ comme le montre la figure 1.

\section{Changement}

de schème

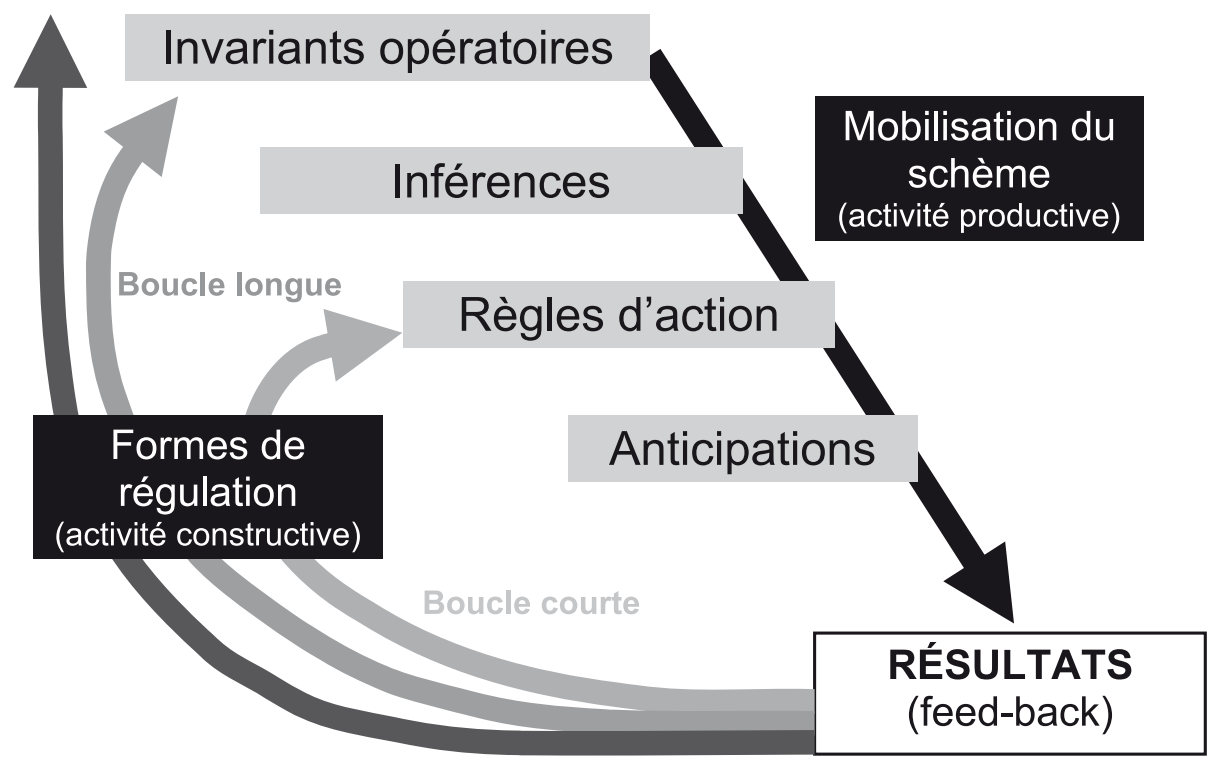

Fig. 1 - Modèle de la compétence

Conformément à la définition présentée ci-dessus, la compétence y est représentée comme, d'une part, la mobilisation d'un schème (organisation dynamique de l'activité) comportant quatre composantes (Vergnaud, 1990) : des invariants opératoires, des inférences, des règles d'action et des anticipations. Les invariants opératoires correspondent à des conceptualisations fondées, pour Vergnaud, sur ce que le sujet, à tort ou à raison, tient pour vrai (théorèmes-en-acte) et pour pertinent (concepts-en-acte). Les inférences assurent les ajustements du schème aux caractéristiques spécifiques de la situation et de la tâche. Quant aux règles d'action, elles donnent lieu à la production d'un résultat que les anticipations permettent de prévoir, en lien avec le but de l'activité. Cette mobilisation de schème correspond à ce que Samurçay \& Rabardel (2004) désignent comme une " activité productive ". Mais, pour eux, cette activité productive s'accompagne toujours d'une " activité constructive ", c'est-à-dire d'une transformation du sujet lui-même sous l'effet de sa propre activité. Ainsi, d'autre part, à partir des feed-back engendrés par la mobilisation d'un schème (ou fournis au sujet par un tiers), peuvent se mettre en place trois formes de régulation : des régulations " en boucle courte " visant les règles d'action du schème mobilisé, des régulations " en boucle longue " visant les invariants opératoires associés à ce schème et des régulations relatives au schème lui-même (changement de schème). Par exemple, si le résultat obtenu par le sujet

12 Cf. Coulet (à paraître) pour la dernière version de ce modèle et ses justifications théoriques. 
diffère de celui qu'il anticipait, une régulation en boucle courte visera à changer de règle d'action, une régulation en boucle longue visera à changer ce que le sujet tenait pour vrai et/ou pour pertinent dans cette situation, quant à une régulation de type changement de schème, elle visera à mobiliser une autre organisation de l'activité, un autre schème. Ce sont ces régulations qui permettent au sujet d'apprendre des situations et des tâches qu'il traite et qui, de ce fait, constituent un élément essentiel pour comprendre la portée de ces acquisitions pour le sujet : un changement de conceptualisation ayant un potentiel adaptatif beaucoup plus large qu'un simple changement de procédure locale même si, bien entendu, celle-ci s'avère cruciale, notamment pour réussir la tâche.

À partir de cette modélisation de la compétence, nous avons considéré que les propos que l'enseignant adresse à ses élèves, au cours des séances de classe pour susciter et accompagner leur activité sur les tâches qu'il leur propose, peuvent concerner différents éléments. Ainsi, par exemple, lorsque l'enseignant dit : " Nous, on va vérifier qu'il y a bien NEUF étoiles sur le sapin ", peut-on considérer que c'est la tâche assignée aux élèves qui est visée par cet énoncé ? De même, a-t-on affaire à une orientation vers un feed-back (par exemple, lorsque, suite à une réponse d'élève, l'enseignant dit : "On est d'accord ") ou à une anticipation quand l'enseignant demande : "Quels sont ceux qui pensent que ça va être une solution JUSTE, exacte, bonne?". La figure 2 représente alors, en référence au modèle de la compétence présenté ci-dessus, tous les types d'interventions que l'enseignant peut adopter dans le guidage de l'activité de ses élèves.

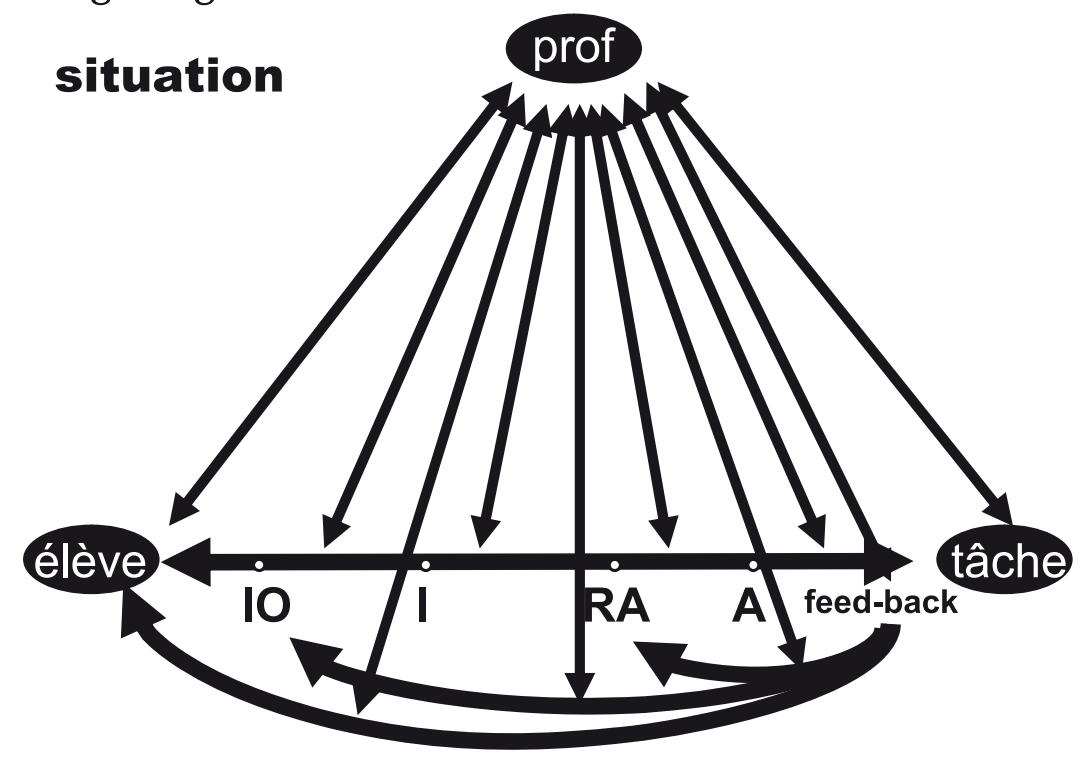

Fig. 2 - Modèle de guidage du développement des compétences des élèves ${ }^{13}$

13 Cf. Coulet (à paraître) pour la présentation de ce modèle sous sa forme générale MADIC (Modèle d'Aide au Développement des Compétences Individuelles). Sur la figure 2, I0 signifie "Invariants Opératoires ", I "Inférences ", RA " Règles d'Action ", A " Anticipations ". Les flèches du bas représentent les trois formes de régulation possibles pour l'élève, à partir des feed-back qu'il obtient concernant son activité sur la tâche. Quant aux flèches verticales et obliques, elles marquent différents schèmes de prises d'information et d'interventions de l'enseignant sur l'activité de l'élève. 
Ainsi, grâce à ce modèle et dans la logique des exemples présentés ci-dessus, nous avons pu catégoriser l'ensemble des propos adressés aux élèves durant les deux séances observées ${ }^{14}$. Nous avons ensuite caractérisé le profil (schèmes de guidage privilégiés) de chacun des deux enseignants observés, dans leur façon de guider le développement des compétences de leurs élèves, en catégorisant, d'abord, les propos qu'ils leur adressent et en les quantifiant ensuite (volume exprimé en nombre de signes dans les transcriptions). La figure 3 présente ces résultats.

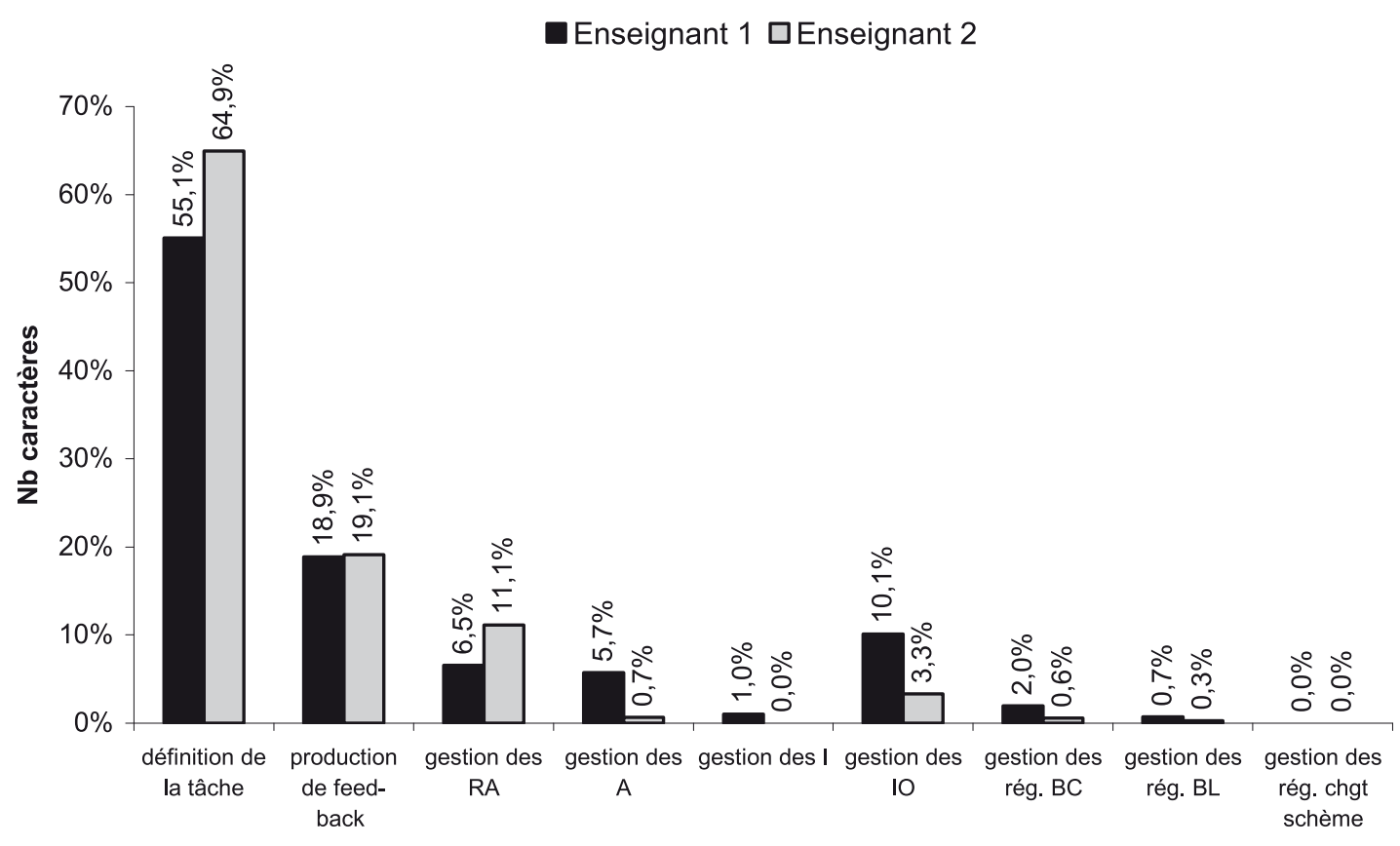

Fig. 3 - Distribution (en \% et pour chaque enseignant) du volume des propos relevant de chaque catégorie d'intervention ${ }^{15}$.

Malgré quelques différences entre les deux enseignants, non commentées ici ${ }^{16}$, la figure 3 révèle surtout une grande similitude dans l'importance qu'ils donnent, tous deux, à certains schèmes de guidage. Ainsi, les schèmes "définition de tâches " et " production de feed-back ", représentent, ensemble, à peu près $80 \%$ de leurs interventions. On peut alors considérer que l'essentiel de l'interaction se déroule comme si les élèves, sollicités par les tâches proposées, voyaient leurs réponses positivement ou négativement renforcées, sur un modèle d'apprentissage relevant de la plus pure tradition béhavioriste.

De façon symétrique, les autres catégories d'interventions représentent une part assez faible, environ 20 \% au total. Parmi elles, le schème " gestion des invariants

\footnotetext{
14 Les séances étaient vidéoscopées et, ensuite, entièrement retranscrites.

15 De gauche à droite sur la fig. 3, ces catégories sont : définition de la tâche, production de feed-back, gestion des règles d'action, gestion des anticipations, gestion des inférences, gestion des invariants opératoires, gestion des régulations en boucle courte, gestion des régulations en boucle longue, gestion des régulations de type changement de schème.

16 Pour une analyse différentielle des deux profils, voir Coulet (2007b).
} 
opératoires " n'est pas (surtout pour l'un des deux enseignants) aussi mobilisé qu'on aurait pu l'attendre, faisant, au mieux, jeu égal avec le schème "gestion des règles d'action ". Ces données suggèrent que la focalisation des enseignants sur les formes de conceptualisation de leurs élèves reste très modeste au regard de l'ensemble de leurs interventions. De la même manière, on constate que le volume des interventions est également très faible en ce qui concerne les schèmes " gestion des inférences " ou " gestion des régulations ". Là encore, tout se passe comme si le guidage de l'activité des élèves appelait peu de propos relatifs à ses variations (en fonction des tâches ou des résultats obtenus), au bénéfice de la prise en compte de son caractère prototypique, c'est-à-dire de tout ce qui renvoie à une démarche canonique, permettant de produire la bonne réponse.

Plus généralement, ces résultats peuvent être interprétés comme une propension chez ces enseignants, dans la réalité de leur activité (sans doute, à leur insu et, probablement même, en contradiction avec leurs conceptions pédagogiques explicites !), à privilégier un guidage focalisé sur la réussite des tâches par les élèves, au détriment d'autres formes de guidage plus directement en lien avec la compréhension, c'est-à-dire avec les différentes formes de conceptualisation mises en jeu par les élèves. Autrement dit, une analyse fine des pratiques de ces deux enseignants, semble révéler une tendance à orienter leurs interventions vers une gestion très déséquilibrée du " réussir " et du " comprendre " qui peut être mise en relation avec les résultats des élèves dans la comparaison entre pré-test et posttest. Même s'il convient de rester prudent sur ces données pour diverses raisons techniques ${ }^{17}$, on note, malgré tout, que la réussite aux épreuves de généralisation (tâches différentes de celles qui ont été utilisées durant les deux séances de classe) proposées en post-test, reste assez faible. Les élèves tirent donc bien profit du guidage qui leur a été proposé pour réussir les tâches qu'ils ont eues à traiter durant leur apprentissage mais, semble-t-il, sans en dégager les principes sous-jacents, susceptibles d'engendrer le traitement efficace d'une classe de tâches plus étendue.

Évidemment, ces résultats, au-delà même de la portée qu'ils peuvent avoir, compte tenu notamment de la spécificité des situations étudiées et du danger qu'il y aurait à généraliser trop rapidement, constituent néanmoins une sérieuse invitation à réfléchir sur ce qui se joue ou peut se jouer (l'hypothèse n'est pas à exclure), pour les enseignants et les élèves, dans le processus d'enseignement-apprentissage, tel qu'il se déroule en classe.

17 II s'agit, notamment de problèmes d'effectifs, du choix et du nombre d'épreuves, de distribution des réussites et échecs au pré-test. 


\section{Attendus et malentendus dans le processus enseignement/apprentissage}

En effet, les résultats de cette étude qui concerne, rappelons-le, deux maîtres formateurs, nous conduisent à nous demander si l'acquisition d'une notion n'est pas implicitement conçue par les enseignants (et, probablement, par voie de conséquence, par les élèves eux-mêmes, voire par de nombreux parents d'élèves) comme relevant essentiellement de la réussite à un certain nombre de tâches. Par ailleurs, et pour reprendre notre réflexion sur la référentialisation (cf. BO, $\mathrm{n}^{\circ} 29$ du 20 juillet 2006 et BO, n 1 du 4 janvier 2007), il faut bien reconnaître que la définition des compétences à travers des listes de connaissances, capacités et attitudes, exprimées uniquement sous la forme d'un état final, comme but à atteindre, constitue également une injonction forte à la focalisation des différents acteurs sur le résultat à obtenir, beaucoup plus que sur les processus en jeu dans l'évolution visée. Or, cette propension à privilégier le versant " réussite " de l'activité, au détriment des processus qui l'engendrent pose deux problèmes essentiels.

En premier lieu, comme le soulignent Bastien et Bastien-Toniazzo (2004), le recouvrement entre réussite et compréhension est loin d'être parfait. Ainsi, par exemple, un élève peut fort bien réussir un grand nombre de problèmes arithmétiques en se fondant sur une conception erronée du type : "il faut faire une addition si l'on voit le mot "plus" dans l'énoncé et une soustraction si c'est le mot "moins" que l'on y trouve ". On comprend alors combien peut être grand le malentendu dans l'interaction maître-élève, tant que cette conception erronée de l'élève n'a pas été débusquée comme organisatrice de son activité sur ce type de tâches. On saisit surtout à quel point un guidage des apprentissages, essentiellement constitué de tâches à résoudre et de feed-back fournis sur les résultats obtenus, peut s'avérer contre-productif.

En second lieu, une trop grande focalisation sur le résultat final risque fort de conduire à développer des stratégies pédagogiques visant d'emblée ce but, quitte à en passer par un étayage maximal (pouvant aller jusqu'à " l'effet Topaze "18) pour assurer la réussite de la tâche, plutôt que de concevoir des étapes d'acquisition à partir de l'analyse des caractéristiques des compétences actuelles des élèves.

On se prend alors à rêver de référentiels qui ne se limitent pas à souligner comme, par exemple pour le socle commun à propos des mathématiques : "La maîtrise des principaux éléments de mathématiques s'acquiert et s'exerce essentiellement par la résolution de problèmes, notamment à partir de situations proches de la réalité " (B0, n 29 du 20 juillet 2006, p. 7), mais s'attachant également à attirer l'attention des maîtres sur les processus de résolution et de conceptualisation mis en jeu par les élèves pour traiter les tâches qu'on leur propose. Bien sûr, la définition des

18 L'enseignant, à la façon de Topaze, le célèbre personnage de Pagnol, suggère alors la réponse attendue en la dissimulant sous des formes de plus en plus transparentes. 
compétences professionnelles des maîtres (BO, $\mathrm{n}^{0} 1$ du 4 janvier 2007) souligne bien, parmi les capacités liées à la compétence " concevoir et mettre en œuvre son enseignement ", que :

- "Le professeur est capable [...]

- de raisonner en termes de compétences, c'est-à-dire déterminer les étapes nécessaires à l'acquisition progressive des connaissances, des capacités et des attitudes prescrites à partir des acquis et des besoins identifiés en mettant en œuvre : une progression et une programmation sur l'année et sur le cycle ; une progression différenciée selon les niveaux des élèves ;

- de s'appuyer sur ses connaissances des processus d'apprentissage des élèves et de la psychologie de l'enfant, de l'adolescent et du jeune adulte ;

- de prendre en compte les résultats des évaluations dans la construction d'une progression pédagogique".

Toutefois, exprimées à un tel niveau de généralité et toujours en référence à la distinction entre connaissances, capacités et attitudes, on peut se demander dans quelle mesure, ces prescriptions peuvent véritablement focaliser l'attention des enseignants sur les processus de traitement des tâches par les élèves et, partant de là, de les orienter dans la conception et la mise en œuvre d'une médiation ou d'une remédiation adaptée.

Au total, les référentiels fondant l'approche par compétence à l'école, s'ils offrent un grand nombre de propositions utiles quant à l'explicitation des attentes en matière de " socle commun " et de " compétences professionnelles des enseignants ", se révèlent néanmoins porteurs de caractéristiques moins positives quant à leur capacité à générer des pratiques en accord avec les objectifs visés. Dès lors, quelles solutions peut-on imaginer pour éviter ces écueils?

\section{Au-delà de la prescription : des référentiels ouverts et mutualisés}

Parmi les pistes envisageables, il nous semble tout d'abord indispensable de rompre avec une définition statique, taxinomique, de la compétence dont la trilogie des savoirs, savoir-faire et savoir-être ne peut exprimer les processus qu'elle met en œuvre dans la confrontation située d'un sujet avec une tâche donnée.

En second lieu, même si la norme de référence, en termes de résultats attendus, reste incontournable dans une telle approche référentielle, celle-ci ne devrait pas masquer la nécessité d'une focalisation sur les voies empruntées pour parvenir à ce résultat. À ce niveau, on ne soulignera jamais assez l'importance cruciale d'un diagnostic fin de ce que sont les caractéristiques de la compétence initialement mobilisée par le sujet sur la tâche pour, sur cette base précise, concevoir une médiation ou une remédiation adaptée. 
Enfin, concernant ce guidage par l'enseignant de la construction des compétences des élèves, on ne peut qu'affirmer avec force la nécessité de l'organiser afin d'instaurer une dialectique maîtrisée du "réussir " et du " comprendre " en résistant à la tentation de considérer la présence du premier comme une preuve suffisante de la construction du second.

Dans cette perspective, au-delà de leur caractère normatif, les référentiels devraient pouvoir évoluer vers des instruments ${ }^{19}$ (Rabardel, 1995) que les enseignants s'approprient pour y capitaliser leur expérience professionnelle. Ainsi, par exemple, à telle compétence à construire devrait pouvoir être associés des descriptifs de compétences initiales ou intermédiaires, repérées chez les élèves (formes de conceptualisation mobilisées sur telle ou telle tâche, procédures de résolution de tel ou tel type, etc.). De la même manière et parallèlement, pourraient y être consignées des modalités de guidage testées comme particulièrement efficaces ou, au contraire, à éviter pour telle ou telle raison, etc. Quant à l'ensemble de ces traces, tirées de l'expérience professionnelle des contributeurs à des référentiels mutualisés et, donc, effectivement représentatifs d'une communauté professionnelle, elles pourraient être systématiquement organisées ${ }^{20}$ selon des rubriques déduites d'un modèle théorique de la compétence et des activités de tutelle (cf. ceux que nous avons présentés plus haut).

On peut alors espérer que des instruments ainsi conçus puissent, à la fois, permettre une mutualisation de nombreuses expertises professionnelles, jusqu'ici peu exploitées ${ }^{21}$ et susciter d'importantes restructurations des pratiques, bien au-delà des controverses portées par les tenants ou adversaires de l'APC.

\section{Conclusion}

Seul un modèle théorique et fonctionnel de la compétence peut permettre la mise en perspective des controverses soulevées par l'APC et, en référence à un tel cadre, de penser, de façon cohérente et systématique, le guidage des apprentissages des élèves à travers des référentiels de compétence d'une toute autre nature. II s'agirait, alors, de rompre avec une logique taxinomique, par trop dogmatique et favorisant une focalisation sur le résultat à atteindre au bénéfice d'une capitalisation de l'expérience pédagogique de la communauté enseignante, ouverte sur la dialectique du "réussir " et du " comprendre ", et sur l'innovation.

Jean-Claude COULET

jean-claude.coulet@uhb.fr

19 Pour Rabardel, un instrument est une entité mixte comportant, à la fois, un artéfact (objet matériel ou symbolique généré dans une culture donnée) et un ou des schèmes d'utilisation.

20 Pour un exemple de référentiel de compétence de ce type, cf. Coulet, 2007a.

21 Tant il est vrai que l'institution se caractérise avant tout par un fonctionnement hiérarchique, plus favorable à des pratiques d'application par des enseignants isolés que par des démarches de production mutualisées susceptibles de constituer des références largement partagées par une communauté de professionnels. 


\section{BIBLIOGRAPHIE}

BASTIEN C. \& BASTIEN TONIAZZO M. (2004). Apprendre à l'école, Paris : A. Colin.

BELLIER S. (1999). " La compétence ", in P. Carré. \& P. Caspar, Traité des sciences et techniques de la formation, Paris: Dunod, p. 223-244.

CARETTE V. (2007). " Les implications de la notion de compétence sur l'évaluation en Communauté française de Belgique ", Éducation et Formation, e-286, p. 51-61.

CHAUVIGNÉ C. \& COULET J.-C. (2010). "La démarche compétence : nouveau paradigme pour la pédagogie universitaire ? ", Revue française de pédagogie, $\mathrm{n}^{0} 172$ [à paraitre].

COULET J.-C. (à paraître). "La notion de compétence : un modèle pour décrire, évaluer et développer les compétences ", Le Travail Humain.

COULET J.-C. (2007a). " Le concept de schème dans la description et l'analyse des compétences professionnelles: formalisation des pratiques, variabilité des conduites et régulation de l'activité ", in M. Merri, Activité humaine et conceptualisation. Questions à Gérard Vergnaud, Toulouse : PUM, p. 297-306.

COULET J.-C. (2007b). Caractérisation des schèmes des enseignants. Communication présentée au colloque international Les effets des pratiques enseignantes sur les apprentissages des élèves, Besançon, France, 14 et 15 mars.

CRAHAY M. (2006). "Dangers, incertitudes et incomplétude de la logique de la compétence en éducation ", Revue française de pédagogie, n 154, p. 97-110.

FABRE M. (1997). "Pensée pédagogique et modèles philosophiques : le cas de la situation-problème. ", Revue française de pédagogie, nº 120, p. 49-58.

GAUDRON J.-P. \& CROITY-BELZ S. (2005). "Bilan de compétences (BC) : états des recherches sur les processus psychologiques en jeu ", Psychologie du travail et des organisations, $\mathrm{n}^{0} 11$, p. 101-114.

HIRTT N. (2009). "L'approche par compétences : une mystification pédagogique ", L'école démocratique, $\mathrm{n}^{0}$ 39, p. 1-34.

JONNAERT P. (2006). "Action et compétence, situation et problématisation ", in M. Fabre et E. Vellas, Situations de formation et problématisation, Bruxelles : De Boeck, p. 31-40

LENOIR Y. \& PASTRÉ P. (2008). Didactique professionnelle et didactiques disciplinaires en débat : un enjeux pour la professionnalisation des enseignants, Toulouse : Octarès.

LICHTENBERGER Y. (1999). "La compétence comme prise de responsabilité ", in Entreprises et compétences: le sens des évolutions, Paris : Les cahiers des clubs CRIN, p. 69-85.

OIRY E. (2005). "Qualification et compétence : deux sœurs jumelles? ", Revue Française de Gestion, vol. 5, $\mathrm{n}^{0}$ 158, p. 13-34. 
MONCHATRE S. (2008). L'“ approche par compétence ", technologie de rationalisation pédagogique. Le cas de la formation professionnelle au Québec, Net. Doc, $\mathrm{n}^{\circ} 36$, p. 1-55, disponible sur Internet : < http://www.cereq.fr/pdf/Net-Doc-36.pdf>, consulté le 25 août 2010.

PERRENOUD P. (1999). Dix nouvelles compétences pour enseigner; invitation au voyage, Paris: ESF.

PERRENOUD, P. (2002). " Apprendre à l'école à travers des projets : pourquoi ? comment?", Éducateur, n 14, p. 6-11.

PIAGET, J. (1974). Réussir et comprendre, Paris : PUF.

RABARDEL P. (1995). Les hommes et les technologies : approche cognitive des instruments contemporains, Paris: A. Colin.

SAMURÇAY R. \& RABARDEL P. (2004). "Modèles pour l'analyse de l'activité et des compétences, propositions ", in R. Samurçay \& P. Pastré, Recherches en didactique professionnelle, Toulouse : Octarès, p. 163-180.

SAVOYANT A. (1999). "Compétence, performance et activités", in Entreprises et compétences : le sens des évolutions, Paris : Les cahiers des clubs CRIN, p. 179-191.

SENSEVY G. (2007). Caractérisation des pratiques d'enseignement et détermination de leur efficacité. La lecture et les mathématiques au cours préparatoire. Rapport de recherche, Programme incitatif de recherche en éducation et formation (PIREF).

STROOBANTS M. (1998). "La production flexible des aptitudes ", Éducation permanente, $\mathrm{n}^{\circ} 135$, p. 11-21.

VERGNAUD G. (1990). " La théorie des champs conceptuels ", Recherche en didactique des mathématiques, vol. 10, $n^{\circ}$ 2/3, p. 133-170.

\section{Abstracts • Zusammenfassungen $・$ Resúmenes}

\section{The referencialization of competences in schools, conception and implementation: from avenues to misunderstandings}

Starting from a research analyzing some teachers' activity on the basis of a functional model of competence and beyond the debate over the competence-based approach (APC), this paper aims to show how the way the French Ministry of Education's reference systems are drawn up may well intensify certain misunderstandings in the field of the dialectics of "achievement" and "understanding" in the pupils' learning processes. A few avenues of reflection are then proposed with a view to making use of these systems of reference beyond their normative aspect, for the sake of a building-up of teachers' professional skills. 


\section{Die Referentialisierung der Kompetenzen in der Schule, Auffassungen und Anwendungen: von den Erwartungen bis zu den Missverständnissen}

Anhand einer Forschungsarbeit, die dieTätigkeit der Lehrer nach einem funktionellen Modell der Kompetenz analysiert, zielt der Artikel darauf ab, über die von dem kompetenzorientierten Ansatz („APC”) ausgelösten Debatten hinaus zu zeigen, dass die Art und Weise, wie das französische Erziehungsministerium die Bezugssysteme formuliert, gewisse Missverständnisse, die die Dialektik des „Reüssierens” und des „Verstehens” beim Lernen betreffen, zu akzentuieren droht. Einige Überlegungspisten werden dann vorgeschlagen, um diese Bezugssysteme über ihren normativen Aspekt hinaus im Dienste einer Kapitalisierung der Berufskompetenzen der Lehrer zu instrumentalisieren.

\section{La referencialización de las competencias en la escuela, concepciones y puestas en práctica: de las expectativas a los malentendidos}

A partir de una investigación que analiza la actividad de profesores sobre la base de un modelo funcional de la competencia, este articulo propone mostrar, más allá de los debates suscitados por el acercamiento por competencias (APC), de qué manera la formulación de los referenciales del ministerio de educación francés corre el riesgo de acentuar ciertos malentendidos relativos à la dialéctica del " aprobar " y del " comprender " en el aprendizaje de los alumnos. A continuación proponemos algunas pistas de reflexión con vista a instrumentalizar dichos referenciales, más allá de su aspecto normativo, al servicio de una capitalización de competencias profesionales de los docentes. 
